\title{
A RARE CASE REPORT OF EVANS SYNDROIME IN PREGNANCY
}

KEY WORDS: Evans

syndrome, Autoimmune Hemolytic anemia, Direct Antiglobulin Test, Idiopathic Thrombocytopenic Purpura.

\section{Dr.T. Susmitha*}

\section{Dr.G. Nirmala} Vani
M.S OBG Assistant Professor, Department of Obstetrics \& Gynaecology, GGH, Guntur, Andhra Pradesh*Corresponding Author

M.S OBG Post Graduate, Department of Obstetrics \& Gynaecology, GGH, Guntur, Andhra Pradesh.

The simultaneous or sequential development of autoimmune hemolytic anemia (AIHA) and idiopathic thrombocytopenic purpura (ITP) is known as Evans syndrome, which is very rare in pregnancy. We experienced a case of Evans syndrome in pregnancy. A 23-year-old primigravida with 37 weeks of gestation presented to the labor room with Evans syndrome. Her blood reports revealed features of hemolysis with macrocytic anemia, thrombocytopenia, elevated LDH, and low serum haptoglobin levels, and a direct antiglobulin test (DAT) was positive. A healthy female newborn with $3200 \mathrm{~g}$ was delivered by cesarean section. After delivery, DAT remained positive, and her platelet count falls markedly, and bone marrow transplantation was done. When Evans syndrome diagnosed during pregnancy, close and careful observation is essential because it can worsen both the maternal and fetal condition.

\section{INTRODUCTION}

In a pregnant woman, hemoglobin concentration and hematocrit decrease as a result of marked plasma augmentation. Moderate or severe anemia may, however, causes fetal growth restriction or premature birth. Thus, it is important to identify and properly manage the cause of anemia. Hemolytic anemia is one cause of moderate or severe anemia. Evans syndrome is characterized by simultaneous or sequential development of idiopathic thrombocytopenic purpura and autoimmune hemolytic anemia in the absence of a known underlying cause. Autoantibodies against erythrocytes, platelets have been shown in Evans syndrome. It was first described by Robert Evans in 1951. The occurrence of isolated episodes of thrombocytopenia and hemolytic anemia and the results of in vitro studies have suggested the roles of non-cross-reacting autoantibodies targeted at different antigenic determinants on red cells and platelets. Incidence of Evans syndrome is $1.8 \%$ to $10 \%$ of patients with ITP.We experienced a case of Evans syndrome in pregnancy.

\section{CASE REPORT}

A 23-year-old primigravida with 37 weeks of gestation was referred to Government General Hospital, Guntur in view of Evans syndrome. She was a diagnosed case of Evans syndrome in her 5 th month of gestation. Since then, she was on oral prednisolone $40 \mathrm{mg}$ daily. It was her planned pregnancy after ovulation induction, and she had regular antenatal checkups. She presented with anemia and thrombo cytopenia, and the direct antiglobulin test was positive. On physical examination, her blood pressure was $130 / 80 \mathrm{~mm}$ of Hg; Pulse rate was $84 / \mathrm{min}$, Respiratory rate was $16 / \mathrm{min}$. Her height and weight were $145 \mathrm{~cm}$ and $52 \mathrm{~kg}$, respectively. Uterus correspond to term gestation.

Blood tests included a hemoglobin of $7.8 \mathrm{~g} / \mathrm{dL}$, hematocrit of $24.8 \%$, white cell count of 6000 cells/cumm with $80 \%$ neutrophils. The platelet count was 63,000/ $\mathrm{mm} 3$. Prothrombin time and activated partial thromboplastin time were normal. The blood group was $\mathrm{A}+\mathrm{ve}$. Liver function and renal function tests were normal. Peripheral smear showed microcytic hypochromic anemia with no schistocytes. ANA was negative. LDH was $922 \mathrm{U} / \mathrm{L}$; Serum Haptoglobin was $<5$ $\mathrm{ng} / \mathrm{ml}$. Vitamin B12 \& Serum Folate levels were normal. The direct antiglobulin test was positive, and the Indirect Antiglobulin test was negative. The lupus anticoagulant test was negative. Her glucose challenge test was normal. The thyroid profile was normal. A complete urine examination was normal. Ultrasound revealed a single viable fetus of 37 to
38 weeks gestation with normal doppler changes.

In view of anemia, she was given 1 unit of packed cell transfusion two days after admission. Later she developed hematuria, and her platelet count reduced to $40,000 / \mathrm{cumm}$. Then she was given four units of platelet concentrate transfusion. She was given intravenous methylprednisolone to increase the platelet count. She underwent emergency Lower Segment Cesarean Section in view of cephalopelvic disproportion, and a healthy female newborn of $3.2 \mathrm{~kg}$ birth weight was delivered. Her APGAR score was 8 and 9 at $1 \mathrm{~min}$ \& $5 \mathrm{~min}$, respectively. During surgery, she developed atonicity, and as the bleeding was not controlled with oxytocics, stepwise devascularisation was done. Then bleeding was controlled. Intra-operatively she was given two units of packed cells, two units of platelet and two units of FFP. During the postoperative period, her platelet count decreased to 20,000/ 1 , Hb level was $10 \mathrm{~g} / \mathrm{dl}$. Prednisolone $40 \mathrm{mg}$ was started, and platelet concentrates were given. Both the mother and the neonate were discharged on postdelivery day 8. After discharge, she continued on prednisolone, her DAT remained positive. Hemoglobin level was $10.5 \mathrm{~g} / \mathrm{dL}$, and her platelet count was decreased to 32,000 / cumm. She was admitted to the Department of Hematology, and the prednisolone dose was increased to $60 \mathrm{mg}$, and progress was satisfactory.

\section{DISCUSSION}

Evans syndrome is a chronic hematological condition associated with the simultaneous or sequential occurrence of Coombs positive autoimmune hemolytic anemia and idiopathic thrombocytopenic purpura. As with autoimmune hemolytic anemia, Evans syndrome may have an underlying cause, but in this case, there was no underlying disorder found. Evans syndrome is a rare occurrence during pregnancy. In our case, Evans syndrome was diagnosed in the fifth month of the period of gestation.

The two most common causes of anemia during pregnancy and the puerperium are iron deficiency and acute blood loss. Other causes include inflammation, malignancy, megaloblastic anemia, and acquired hemolytic anemia. In our case, acute blood loss, megaloblastic anemia, and malignant diseases were unlikely. Blood tests showed an increase of reticulocytes and LDH levels and a decrease in the haptoglobin level. As a result, an acquired hemolytic disease was suspected because the patient had no history of congenital hemolytic anemia.

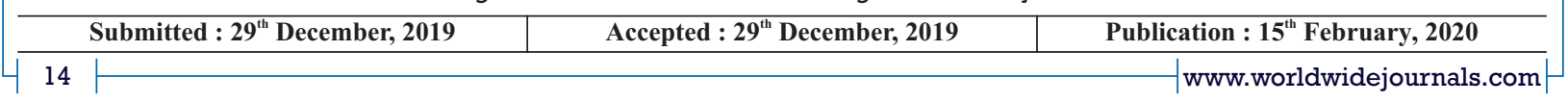


Hemolysis occurs under many conditions, such as HELLP syndrome, acute fatty liver of pregnancy (AFLP), HUS, and TTP, or as a result of medication. In our case, the blood test results and medication history did not correspond to HELLP syndrome, AFLP, HUS, TTP, or drug-induced hemolysis. Furthermore, the DAT was positive, indicating the likelihood of AIHA. AIHA is a disease characterized by the development of anti-erythrocyte autoantibodies and the destruction of erythrocytes. This disease is classified as warm (65\%), cold $(30 \%)$, and mixed $(5 \%)$ type. The main clinical features of AIHA are acute anemia, hemolysis, and a positive DAT result.

Treatment, in our case, was corticosteroids. The immediate outcome of treatment was acceptable. Her platelet count increased after treatment and remained stable throughout the pregnancy. Other treatments used for Evans syndrome include intravenous gamma immunoglobulin, danazol, cyclophosphamide, vinca alkaloids, azathioprine, plasmapheresis, and splenectomy, especially for refractory or recurrent cases.

Complications of pregnancy, in our case, was postpartum hemorrhage. Other reported complications of pregnancy have been hemorrhagic complications such as abruption of placenta and postpartum hemorrhage. Complications in Evans syndrome in pregnancy can be divided into the consequences of autoimmune hemolytic anemia and autoimmune thrombocytopenia. Pregnancy in association with autoimmune hemolytic anemia may provoke life.threatening anemia in $40-50 \%$ of cases, and stillbirth or severe postpartum hemolytic anemia in $35-40 \%$ of infants. In our case, the new born was healthy without any complications. The mode of delivery in pregnant women with Evans syndrome depends on obstetric indications. Hemorrhagic complications have not been shown to be related to the mode of delivery, and cesarean delivery should be reserved for obstetric indications only and requires a multidisciplinary approach involving specialists from obstetrics, neonatology, and hematology.

\section{CONCLUSION}

In conclusion, Evans syndrome in pregnancy is a very rare condition and requires a multidisciplinary approach involving specialists from obstetrics, neonatology, and hematology. Close maternal and fetal surveillance and management during pregnancy is essential to increase the possibility of a favorable pregnancy outcome in these women.

\section{CONFLICTS OF INTEREST}

There are no conflicts of interest

\section{REFERENCES}

1. V. Phupong, W. Sareepapong, and P. Witoonpanich, "Evans syndrome and pregnancy: A case report," BJOG: An International Journal of Obstetrics \& Gynaecology,vol.111,no.3,pp.274-276,2004.

2. Michel M.Warm autoimmune hemolytic anemia sand Evans syndrome in adults[French].Rev Med Interne .2008;29:105-114.

3. Michel M, ChanetV, DechartresA, et al. The spectrum of Evans syndrome in adults: new insight into the disease based on the analysis of 68 cases.Blood .2009;114(15):3167-3172.

4. Norton A, Robert sI. Management of Evans syndrome. Br J Haematol.2006;132:125-137.

5. Kaushansky K, Williams WJ. Williams hematology. New York: McGraw-Hill Medical;2010.

6. G. Ronnenberg, R. J. Wood, X. Wang et al., "Preconception hemoglobin and ferritin concentrations are associated with pregnancy outcome in a prospective cohort of Chinese women," Journal of Nutrition, vol. 134, no. 10, pp. 2586-2591,2004.

7. Mishra,N.Dave, and K.Viradiya,"Fatal anaphylactic reaction to iron sucrose in pregnancy,"'Indian Journal of Pharmacology, vol. 45, no. 1, pp. 93-94,2013

8. J. C. Jaime-Pérez, L. N. Guerra-Leal, O. N. López-Razo, N. Méndez-Ramírez, and D. Gómez-Almaguer, "Experience with Evans syndrome in an academic referral center," Revista Brasileira de Hematologia e Hemoterapia, vol. 37, no. 4,pp. 230-235,2015.

9. S. Issaragrisil and M. Kruatrachue, "An Association of Pregnancy and Autoimmune Haemolytic Anaemia," European Journal of Haematology, vol. 31, no. 1,pp. 63-68, 1983.

10. P. Rai, G. Sharma, D. Singh, and J. Garg, "Rare presentation of mixed autoimmune hemolytic anemia in children: Report of 2 cases," Journal of Laboratory Physicians, vol. 9, no. 4,pp.332-336,2017.

11. F. R. Mauro, F. Trastulli, C. Alessandri et al., "Clinical relevance of silent red blood cell autoantibodies," Haematologica, vol. 102, no. 12, pp. e473-e475, 2017.

12. Hoppe, W. Stibbe, A. Bielefeld, A. Pruss, and A. Salama, "Increased RBC autoantibody production in pregnancy," Transfusion, vol. 41 , no. 12, pp. 1559-1561,2001 